Portland State University

PDXScholar

$11-22-2019$

\title{
Attachment in Higher Education and the Workplace: A Literature Review
}

\author{
Emily A. Robey \\ Portland State University
}

Follow this and additional works at: https://pdxscholar.library.pdx.edu/honorstheses

\section{Let us know how access to this document benefits you.}

\section{Recommended Citation}

Robey, Emily A., "Attachment in Higher Education and the Workplace: A Literature Review" (2019). University Honors Theses. Paper 801.

https://doi.org/10.15760/honors.819

This Thesis is brought to you for free and open access. It has been accepted for inclusion in University Honors Theses by an authorized administrator of PDXScholar. Please contact us if we can make this document more accessible: pdxscholar@pdx.edu. 
Attachment in Higher Education and the Workplace: A Literature Review

Emily Robey

Portland State University 


\section{Introduction}

Attachment orientation is most often studied in the context of parent-child and romantic relationships. Recent research begins to delve deeper into the complex workings of our attachment systems by exploring the implications of specific attachment orientations in environments not traditionally associated with attachment behavior such as higher education and the workplace. This exploration is crucially important to understanding the potentially far-reaching effects that attachment theory has on all parts of life and society. Additionally, understanding the influence of attachment on all aspects of life will help us to understand the experiences, perceptions, well-being, and success of individuals in a variety of contexts.

The focus of this literature review is on higher education and workplace environments for a number of reasons. First, there is a significant lack of attachment research in higher education. With college being a transitional period in life the likelihood of attachment orientation changing, or being influenced is likely higher than at other times in life (Hiester, Nordstrom \& Swenson 2009). Additionally, in both the workplace and higher education environments individuals are often in leader-follower relationships that mimic parent-child relationships. Research shows that these kinds of relationships may hold more weight in the influence of attachment related behaviors and shifts in attachment than other relationship dynamics (Keller \& Cacioppe 2001). Finally, people spend a large portion of their lives at work with research showing connections between work satisfaction and life satisfaction (Judge \& Watanabe, 1993) pointing to important influences of work on overall well being.

To truly understand how attachment affects all aspects of life we must also start looking through a lens that takes into account the influence of context on the way that attachment 
behaviors manifest themselves. As such, this literature review will explore two areas of interest for non-traditional attachment research: higher education and the workplace. This exploration will eventually culminate in a discussion of how these studies add to the current attachment literature and the potential implications for future research.

\section{Classical Attachment}

Classical attachment theory, originated in studies of the relationship between infants and mothers, as is the case in the work of early theorists John Bowlby and Mary Ainsworth (Ainsworth, 1989; Bretherton, 1993). Attachment theory proposes a system, or internal working model within infants that allows them to keep caregivers near. Eventually, this working model was shown to allow older infants to attach to important caregiving figures, such as mothers and fathers (Ainsworth, 1989). Bowlby (1958) describes attachment as "the nature of the child's tie to his mother", but Ainsworth and other researchers describe the ability of children to attach to attachment surrogates (Ainsworth, 1989) which include, but are not limited to fathers, siblings, grandparents and teachers. The ability to attach to surrogates stresses the salience of important adult and non-adult figures in children's lives and even later on through adolescents and adulthood.

Classical attachment divides attachment into three distinct categories, or styles: secure, anxious-resistant, and avoidant. These attachment styles develop based on the availability of a secure and responsive attachment figure from the time the child is born throughout their childhood (Ainsworth, 1989; Bowlby, 1958). Children with a secure attachment style are likely to seek out their attachment figures in times of stress, can be easily comforted by these 
attachment figures and respond warmly to the return of an attachment figure (as cited by Bartholomew \& Horowitz, 1991). Anxious-resistant children will act as if they have no interest in their attachment figure while in the same room and will be upset and unable to be comforted after an attachment figure returns (Bartholomew \& Horowitz, 1991). A child with an avoidant attachment style will avoid being close to their attachment figure and will not interact with them when they return after an absence (Bartholomew \& Horowitz, 1991).

\section{Adult Attachment}

More recent research shows that the attachment styles that children develop follow them throughout their lives and are further applied to both romantic and non-romantic relationships (Bartholomew \& Horrowitz, 1991). Attachment is stable, but plastic, meaning that the attachment style that is developed in childhood tends to remain the same into adulthood. Although generally stable, recent studies highlight how life events, both romantic and non-romantic relationships and environmental influences can influence a shift in attachment style in the short and long-term (Brussoni, Jang, Livesley, \& Macbeth, 2000; Davila, Burge \& Hammen, 1997; Davila, \& Sargent, 2003). This section reviews key studies addressing adult attachment.

Adult attachment is classified similarly to classical attachment, but the categories have been broken down even further and the names are slightly different. There are a variety of names for the different adult attachment styles, but for the purpose of this literature review Bartholomew \& Horowitz (1991) classifications will be used to explain the different classifications. These classifications include secure, preoccupied, dismissive and fearful. 
Secure individuals are thought to make friends easily, enter into romantic relationships with ease, feel comfortable with intimacy and closeness, trust others, value relationships as an important and essential part of their well-being and lives and generally show stability in their social and emotional lives (Ravitz, Maunder, Hunter, Sthankiya, \& Lancee, 2009; Bartholomew \& Horowitz, 1991; Ognibene \& Collins, 1998; Vogel \& Wei, 2005). When compared to individuals with insecure attachment orientations, an individual with a secure attachment style has been shown to have a history of better developmental experiences, more personal and interpersonal skills and more trust within their relationships (Lopez, 1997).

Adults with a preoccupied attachment style want to be in close relationships, but often feel anxiety around these relationships related to fears of loss, deception, or some other potential break in attachment (Bartholomew \& Horrowitz, 1991). Preoccupied adults view themselves as distressed, but view others as supportive outlining a mismatch between self concept and concept of others (Kobak and Sceery, 1988). Researchers have also found that preoccupied individuals report more self doubt, less acceptance from other people, higher self awareness in the presence of others and increased self blame when rejected by others (Bartholomew \& Horrowitz, 1991).

A dismissive attachment style is associated with interpersonal problems related to a lack of warmth in relationships (Bartholomew \& Harrowitz, 1991). These individuals tend to maintain their self esteem by being dismissive of the importance of other people in their lives (Bartholomew \& Harrowitz, 1991). To this end, people with a dismissive attachment style tend to view others as unsupportive and untrustworthy (Kobak \& Sceery, 1988; Bartholomew \& Harrowitz, 1991). Bartholomew and Harrowitz (1991) found that dismissive adults generally have trouble getting close to and relying on others. 
Individuals with a fearful attachment style report more interpersonal problems, often times due to their overlying passive way of interacting with others (Bartholomew \& Harrowitz, 1991). Like dismissive attachment, a fearful attachment style is often associated with having more trouble getting close to and relying on others along with being more fearful and less assertive when compared to secure individuals (Bartholomew \& Harrowitz, 1991). Much like preoccupied adults, fearful adults report more self doubt and and less acceptance by other people (Bartholomew \& Harrowitz, 1991).

A study done by Lopez and Gromley (2002) on attachment stability in first-year college students showed only moderate stability in attachment styles over the first year of college outlining the potential of attachment orientation to change, especially during transitional periods. A 23 year long, longitudinal study following 170 children into young adulthood found that some of the children who had originally been classified as insecurely attached by their mothers self-classified themselves as securely attached at ages 20 and 21 (Roisman, Padron, Sroufe \& Engeland, 2002). This phenomena of attachment styles changing from insecure to secure is known in the research community as earned security. It is speculated to be scaffolded by both past and present, long-standing, developmental support that has allowed insecure individuals to overcome parenting styles that correlate to insecure attachment styles (Roisman, Padron, Sroufe \& Engeland, 2002). When viewed through the lense of Ainsworth's idea of attachment surrogates this study suggests an influence outside of the nuclear family on attachment orientation. 


\section{Higher Education}

With recent research showing that insecure attachment orientations are on the rise for American college students (Konrath et al., 2014), it is more important than ever to look at the potential interaction of attachment orientation and higher education. The literature suggests that attachment style influences the way that students interact with the academic environment, seek help, perceive themselves as learners, and create connections such as friendships in school.

Lopez (1997) found that students with an insecure attachment style to their professors were more prone to academic performance-related anxiety, were less likely to pursue challenging coursework, and had weaker feelings of identification and involvement with the university community. In contrast, students with a secure attachment style towards their professors were more likely to endorse mastery-learning attitudes and feelings of connectedness to their university community (Lopez, 1997). Although not statistically significant Lopez (1997) found that students with an insecure attachment style also reported a lower GPA than students with a secure attachment style.

This study suggests that the attachment orientation of a student to their professor has important implications for student success. This outlines the importance of attachment in dyadic, non-intimate relationships in the academic environment. Additionally, a student's attachment style holds significance outside of the realm of one on one relationships such as connection to community and even the type of tasks that a student decides to take on.

Adding to the findings of Lopez (1997), Larose and colleagues (1999) found that an avoidant attachment style in college students was associated with a negative network orientation. This negative network orientation negatively affects the process of help seeking by changing the 
perception that students have of their academic support system. Students who lean more towards an avoidant attachment orientation were found to have negative cognition in regards to the usefulness of their network in providing help. These negative cognitions were found to decrease the likelihood of help seeking behaviors and personal disclosures to professors. This decrease in help seeking behavior is supported by Nelson and Johnson's (2011) findings that college students with insecure attachment orientations may not be receiving the social support they need to be successful in college.

These findings further support the argument that attachment orientation is a highly important variable in understanding the success of college students. Social support and seeking help from both peers and professors have been found to be crucial to academic success (Tinto, 1990). This starts to approach the way in which attachment orientation fans out to affect multiple areas of an individual's success. Not only are insecurely attached individuals viewing themselves in attachment-specific ways, but they are viewing others in attachment-specific ways which leads to a general effect on the way that they are interacting with their environments.

In a study on group work in undergraduate classrooms, Lavy (2017) found that students with insecure attachment styles had lower socio-emotional functioning, reported lower performance in project groups and lower overall satisfaction with the group's performance. Interestingly, this was true even when the students grades did not reflect lower functioning. This study specifically speaks to the negative view of self that individuals with both avoidant and anxious attachment styles are known to possess, the hypervigilance to rejection that anxiously attached individuals often have and the way in which these traits interact with their overall outcomes. It shows that these qualities of attachment style show up and are salient even 
in non-intimate, group interactions within an academic context. More importantly, this study shows, as much as it can with only self-reports, the potential for attachment orientation to interact with the entire classroom environment.

Parade, Leerkes \& Blankson (2010) examined the effects of parental attachment on the ease of forming friendships in female college students. Their findings show that secure attachment predicted a greater ease in forming friendships for both minority and white students. For minority students secure attachment predicted lower social anxiety and more satisfaction from friendships. Adding to Parade and colleagues findings of differences between minority and white students, Nelson and Johnson (2011) found that minority students and women with insecure attachment styles had a lower GPA than male and/or majority students with insecure attachment styles.

The importance of the above studies are twofold. First, Parade and colleagues show that attachment orientation affects a new variable that the other studies have not examined, forming friendships. This finding continues to expand on the importance of attachment across multiple dimensions in an individual's life. Not only are close relationships affected by attachment, but the potential for new relationships are affected. An additional finding that both studies support is the potential overlap between insecure attachment orientation and minority status. This finding is important as it adds to the potential contextual variables that are affecting or interacting with attachment style in an educational environment.

In a higher education context attachment orientation and attachment related behaviors have critical implications. Instructors and academic advisors alike need to be aware of how attachment orientation interacts with the academic environment from the level of the individual 
student to the entirety of the classroom and beyond. Variables such as academic social integration, community involvement and identification with the college community have been found to be crucial to academic success (Tinto, 1990). Given this, the potential outcomes of the feelings of anxiety, weak feelings of identification and involvement, disconnection from community, decrease in help-seeking behavior and feelings of poor group and general functioning in regards to the academic environment can be expected to create negative academic outcomes for students with insecure attachment orientations. It is not only the students with the insecure attachment orientations that we must be concerned about as the above research shows that the entire classroom environment is affected by interactions of attachment orientations.

\section{Workplace}

In the workplace attachment orientation has been shown to influence workplace cohesion, satisfaction with work, emotional regulation at work and interactions between supervisors and employees. Additionally, attachment orientation is associated with specific leadership styles, perceptions of leadership styles and both actual and perceived overall career success.

Kafetsios, Athanasiadou, and Dimou (2014) found that for supervisors an avoidant attachment orientation was related to more positive work outcomes for subordinates, but for subordinates an avoidant, or anxious attachment style was related negatively to positive affect and job satisfaction. This mismatch in outcomes for supervisors and subordinates with avoidant attachment styles was hypothesized to be caused by societal norms that speak to the way that a leader is meant to behave: distant, unemotional and disconnected. 
The results from this study outline the salience of attachment orientation in the workplace. The reduced lack in job satisfaction that insecure attachment was associated with could help to explain chronic change in jobs, or a lack of career progression. Additionally, the proposed hypothesis that societal norms are interacting with the interpretation of attachment behavior speaks to the potential influence of society and culture on the perception and development, or potential for change of attachment orientation.

In a study focusing on line manager and employee interactions, Crawshaw and Game (2014) found that, in regards to their employers, employees with high attachment avoidance reported not feeling like they had a secure base and low interpersonal trust. These individuals were also found to be less satisfied with career growth opportunities, less likely to engage in internal career development opportunities and were more likely to consider alternative employment options. Additionally, they found that the traits that make for successful line managers are comparable to traits that are associated with a secure base.

This study also looked at organizational trust finding a mediating effect between an avoidant attachment style and career attitudes. The authors suggest that the negative interpersonal expectations associated with avoidant attachment may be transferred onto the organization that an individual works for which could influence the level of confidence one has to explore organizational career opportunities. This lack of confidence could lead to the perception that there is a lack of career growth potential.

This study outlines how attachment behaviors follow an individual through their lives in areas other than intimate relationships. Additionally, it shows how attachment orientation interfaces in a variety of ways that we still do not completely understand. These results continue 
to build on the data that speaks to the importance of understanding how attachment orientation affects every aspect of an individual's life from their leadership style, to career growth and feelings about their current work environment.

Molero, Moriano and Shaver (2013) found connections between transformational leadership and employees' attachment style to their bosses. They found that employees were less likely to have anxious, or avoidant attachment styles towards their bosses when they were practicing transformational leadership. Leadership that was more passive, or avoidant was associated with an increase in employees attachment insecurities. Additionally, transactional leadership was positively associated with measures of anxious attachment in employees. Researchers hypothesized that this style of leadership with its theme of reward and punishment could make employees that trend towards anxious attachment more anxious. Molero and colleagues also found that employees with avoidant attachment were less satisfied with their jobs, perceived their bosses as less effective, put less effort in and identify less strongly with their organizations.

The results of this study speak directly to the importance of understanding how attachment orientations can be shifted by specific work environments. To this end it points to the idea that attachment orientation is not only important on an individual level, such as the attachment orientation an employee brings to work influencing the behaviors of that employee, but on a group level, the employees are being influenced by their managers and peers and vice versa. The results of this study point to a varied and highly contextual interaction of different attachment styles within the workplace such as between managers and employees. The findings associated with individuals with an avoidant attachment style are similar to those found in 
Crawshaw and Game's (2014) study, again suggesting that the attachment orientation that an individual brings with them is still influential in the overall outcome, but it is not the only variable to examine.

A study done by Underwood, Mohr, \& Ross (2016) on university personnel found that leadership styles were influenced by attachment orientation. Faculty with secure attachment orientation were more likely to practice transformational and charismatic leadership styles. Faculty with fearful attachment orientations were found to practice charismatic leadership less often while dismissive styles were more likely to practice Laissez Faire leadership.

This study adds to Molero, Moriano and Shaver (2013) study making another connection between a bosses attachment orientation and their leadership style that they choose. Taken together the studies show a trail of interactions, behaviors and outcomes related to attachment orientations. On the individual level bosses and employees are bringing their attachment styles and related behaviors which influence the way in which they lead, react to leadership, perceive interactions and perceive themselves. These variables lead to a workplace where people are influencing each others attachment related behaviors and orientation. This further strengthens the argument that attachment is highly salient in functioning of the entire work environments while simultaneously being influenced by the environment.

Richards and Schat (2011) found that employees who rated higher on attachment anxiety measures practiced fewer citizenship behaviors, had higher levels of instrumental and emotional support seeking and an increased intention to quit. Employees who rated high in measures of attachment avoidance were also found to be associated with higher levels of instrumental and 
emotional support seeking and greater use of surface acting as a means of regulating emotional display.

The finding that both anxious and avoidant attachment orientations were associated with higher levels of support seeking is inconsistent with previous romantic attachment research. This is a highly interesting discovery as it strongly suggests a potential contextual factor in how attachment behavior is displayed. The results of this study go against the idea that you can assume someone's behavior patterns based on their attachment style. This further expands not only on the importance of attachment in work environments, but the importance of context on attachment. Additionally, it suggests that certain environments put pressure on and in turn, influence an individual's attachment behavior.

Much like in higher education employers, human resources managers and others involved in the supervision, management and overall success of employees need to understand how attachment orientation affects the workplace environment. They need to be aware of not only the individual employees behavior, perceptions and overall success, but how attachment interfaces with every aspect of the workplace. This includes understanding how their own attachment style affects their leadership and employee interactions.

Employees with insecure attachment were commonly found to have lower feelings of trust, satisfaction and identification with their organizations and supervisors. This finding is not surprising given the fact that individuals with insecure attachment show these same feelings in their personal lives. These decreased feelings of trust and identification not only negatively impact an individual's performance and success, but they influence interactions with their peers and employers as shown by Langdon and Wang (2009) in their research on attachment and work 
cohesion in Firefighters. The workplace environment is negatively impacted by these feelings, especially if it is the supervisor displaying them.

This review outlines the impact of attachment on supervisors and other leaders. The implications of insecure leadership brings up the question of how our culture reinforces leadership behaviors that are related to insecure attachment. Although not every study showed that supervisors with insecure attachment always lead to bad outcomes for the employees there is enough evidence of the positive effects of secure leadership that we should be moving away from outdated views of successful leaders being cold, distant and unemotional.

\section{$\underline{\text { Discussion }}$}

The purpose of this literature review was to outline the salience and influence of adult attachment in relationships and environments not traditionally studied by attachment researchers, specifically higher education and workplaces. Additionally, the intent was to approach the possibility that context plays a significant role in attachment-related behaviors, that is to say that much like personality researchers have found with personality traits, attachment-related behaviors can change based on the pressures of the environment, whether physical, cultural, or social, that an individual is in. Finally, the literature was examined for areas in need of clarity and for limitations that leave room for future research.

Drawing from the research discussed in this review, we can start to imagine the real-world implications of insecure attachment in the academic environment. For example, when a student has the perception that they are performing poorly in group projects, as insecure students have been shown to have, they become preoccupied with feeling as if they are failing. 
This does not allow them to take advantage of the benefits of group work. The disconnection from group functioning mixed with less help seeking behavior due to a negative network orientation can only lead students to become further behind in their coursework and more dis-enchanted with their education. Indeed, much, but not all, of the limited literature that does compare GPA and attrition rate to attachment orientation has found that insecure students tend to have lower GPAs and a higher likelihood of attrition (Nelson \& Johnson, 2011; Perine, 1999; Lopez, 1997).

In addition to scenarios such as the one given above, understanding the specific implications of attachment for minority college students is of critical importance given that minority students have specific struggles within higher education including but not limited to cultural mismatch and microaggressions (Chang, Wang, Mancini, Mcgrath-Mahrer \& Orama de Jesus, 2019; Simatele, 2018). The limited research available points to the possibility of the attachment orientation of minority students compounding these unique difficulties.

When considering the impact of microaggressions on individuals including increased anxiety, depressive symptoms and negative affect (Nadal, Griffin, Wong, Hamit \& Rasmus, 2014) the finding that secure attachment predicted lower social anxiety and more satisfaction from friendships for minority students is pertinent. If a student is already in an environment that increases their anxiety it would follow that the addition of an insecure attachment orientation would magnify this anxiety. This rise in anxiety could put increasing pressure on the student in turn magnifying the difficulties that they face in successfully completing college. Additionally, forming friendships and connections in college is important for the success of all students, but given the cultural mismatch that many minority students experience they may not have the same 
social opportunities as majority students (Chapman \& Bhopal, 2019). This discrepancy in social opportunities lends to the increased potential of insecure attachment to significantly add to the struggles of forming connections for minority students.

While considering the implications of attachment orientation on the success of minority college students it is important to keep in mind that attachment theory and the tools used to measure attachment orientation have been developed for and by middle-class, westerners (Keller, 2018). This could skew results for minority students and bias interpretation of these results. More research must be conducted to better understand cultural differences in attachment before assuming that we are properly measuring and interpreting attachment across cultures.

The findings of the above studies could help to further inform educators on why certain students struggle with getting involved in the academic community, do not utilize resources, pursue fewer opportunities such as internships, challenging courses and research and generally struggle in the academic arena. Understanding these barriers could lead to new academic advising, counseling, or even teaching styles to reach students with insecure attachment orientations and help them to succeed not only academically, but in their personal lives as well.

In addition to the implications of attachment in higher education this review makes a clear connection between job satisfaction, job success and attachment orientation. Given that researchers have found that increased job satisfaction correlates to increased life satisfaction (Judge \& Watanabe, 1993) it can be assumed that the work-related difficulties caused at least in part by insecure attachment orientation are having a negative impact on an individual's overall life satisfaction. Improving the work environment and better understanding how to help individuals with insecure attachment in the workplace could significantly improve overall life 
satisfaction. This could specifically apply to the relationship between individuals in leadership positions such as managers and employees due to the potential for leader attachment to both positively and negatively influence employee attachment behavior.

The connection between leadership style and attachment orientation is of great importance, especially when viewed through a cultural lens. Given our current cultural environment there are potentially far-reaching, negative personal effects for the individuals in leadership positions with insecure attachment orientations. For example, given the common cultural expectation of leaders being cold, distant and unemotional an individual with avoidant attachment may succeed at work, but be miserable, or lonely in their personal life because they can not maintain a healthy relationship, or maybe any relationship. Is it ethical for our culture to be supporting behaviors that are harming individuals who are practicing them, especially when we know there are less harmful and equally, if not more successful, ways of being a leader?

By understanding the way in which attachment interfaces in work environments we can begin to better explain work-related stress, dissatisfaction, conflict and lack of career growth. Additionally, we can begin to look at how the culture of work environments influences individuals, especially those with insecure attachment orientations in both supervisor and subordinate positions. These findings could lead to new training protocols, more healthful workplace environments and new standards for what a healthy and successful leader looks like.

In addition to specific, real-life applications of the findings of this literature review context stands out as an important influence on attachment related behavior and orientation. Variables such as minority status, culture, social environment and attachment orientation of individuals in leadership positions such as professors and managers are of crucial importance. 
This review speaks to the fact that individuals may be more likely to act a certain way based on their attachment, but the environment holds significant weight in how they actually behave. Future research must begin to approach specific contextual aspects of attachment, especially within a higher education environment where the research is most lacking.

A specific finding of this review is that within attachment-related outcomes in some contexts, traditional patterns are contradicted. For example, an avoidant attachment orientation in supervisors has positive work outcomes for subordinates, but an avoidant attachment orientation in subordinates is associated with negative outcomes. This raises important questions about the potential for differences in the outcome of attachment behavior depending on the individual, dyad, group and overall environment. This is especially interesting given the research that shows that secure attachment is associated with more positive and successful leadership styles. In sum, future research should address differences in outcomes based on the type of work environment, classroom environment, what is culturally expected in those environments, or other supports that may not have been measured.

An additional finding is that leadership styles were found to be associated with different outcomes for employees regardless of attachment orientation. This shows a clear relationship between the environment and the behavior that is displayed. An individual with a certain attachment orientation may be more likely to act a certain way, but external pressures are pushing them in one direction, or another. In an environment where the leadership is charismatic, or transformational insecure individuals attachment schema may be challenged leading to a shift in attachment orientation and/or attachment related behavior. Another possibility is that insecure individuals begin to mirror, or learn from their bosses behavior leading to a replication of the 
secure behavior. This could be compared to how an insecure individual learns from an attachment surrogate such as a therapist, or secure partner (Ainsworth, 1985).

The influence of a supervisors leadership style, which is itself influenced by attachment, on employees attachment, shows a complex and multi-tiered relationship between attachment style and a successful workplace. The environment that an employee is entering into is just as important as the attachment style, or beliefs about how the world works that they hold. Employees do not simply enter into their workplace and start behaving a certain way in accordance with their attachment style. They are constantly adjusting their behaviors based on the messages that they are getting from others in their workplace and social environment. This seems to be especially true of the interactions between employees and supervisors, or individuals in a leadership role.

Attachment research currently focuses very much on the stability of attachment behaviors based on attachment orientations. Researchers have moved towards viewing attachment on a spectrum which is a step in the right direction. The above data suggests that much like personality researchers (Mischel, 2004) along with numerous socio cultural researchers (Kirschner \& Martin, 2010) have found in their fields, attachment behavior needs to be viewed on a situational, or contextual basis. Environment may play a more significant role in the expression and development of attachment orientation than it is given credit for. Separating attachment behavior from the environment leaves limited room for interpretation of meaning and truly understanding how attachment interfaces with the daily life of an individual. 


\section{$\underline{\text { Additional Considerations }}$}

Both within this review and in regards to attachment theory in general there are limitations that must be discussed. First, attachment theory makes assumptions based from a middle-class, western perspective (Keller, 2018). There is an inherent bias in both the interpretation and measurement of attachment orientation along with how "good" and "bad" attachment is defined. The interpretation of attachment can not be accurate without first understanding the culture in which the person is apart of (Keller, 2018). Attachment researchers must move away from the belief that the western view of attachment and the tools that we have developed to measure it can be applied universally to all groups of people. We must re-examine our current view of attachment theory and work to move towards a more culturally informed system.

Along with the lack of cultural sensitivity within attachment theory the type of instrument used to measure attachment can dramatically affect results. Many of the studies examined in this review utilized different methods of measuring attachment leading to a lack of control when comparing results. When considering qualitative research, the bias of the researcher, especially in regards to cultural background, must be taken into consideration. Compounding effects of other variables that researchers are not aware of, or that were not measured must also always be considered especially given the potential of contextual influence on attachment behaviors.

The general lack of attachment research, especially in higher education is an additional limitation. In this case, research examining the attachment orientations of professors, guidance counselors and other individuals in leadership roles would be especially helpful. A more thorough examination of the attachment orientation of every individual in an environment with 
multiple tools would increase our overall understanding of attachment. For example, researchers should be looking for interactions between professor attachment orientation, student attachment orientation and outcomes. Qualitative research including videos could be utilized more often to understand variables such as how the classroom environment, such as reactions of students and professors to specific behaviors, are interacting with attachment orientation and display of attachment behavior.

To improve the validity of future attachment research investigators should work towards implementing mixed methods research protocols that include not only survey measures, but personal interviews and potentially even researcher interpretation via videos or observation. Additionally, researchers must look beyond the individual and dyadic relationships and towards group interactions. As the above research outlines, viewing attachment from a group perspective gives more in-depth information on what influence different interactions have on attachment as well as the way that different attachment orientations interact with each other.

By critically examining the current literature on attachment in the workplace and higher education environments it is clear that attachment needs to be examined through a different lense and with a different set of tools. Traditionally, attachment orientation has been measured by collecting quantitative data such as surveys, or limited qualitative data such as interviews with an individual, or their family. Moving towards a qualitative based and holistic approach to attachment research is the next step in understanding the complex workings of attachment and how it truly interacts with every part of the human experience. 


\section{$\underline{\text { References }}$}

Ainsworth, M. (1985). Attachments across the life span. Bulletin of the New York Academy of Medicine, 61(9), 792-812.

Ainsworth, M. D. S., Blehar, M. C., Waters, E., Wall, S. N., Blehar, M. C., Waters, E., \& Wall, S. N. (2015). Patterns of Attachment: A Psychological Study of the Strange Situation. https://doi.org/10.4324/9780203758045

Bartholomew, K., \& Horowitz, L. (1991). ATTACHMENT STYLES AMONG YOUNG-ADULTS A TEST OF A 4-CATEGORY MODEL. Journal Of Personality And Social Psychology, 61(2), 226-244. https://doi.org/10.1037/0022-3514.61.2.226

Bretherton, I. (1993). The Origins of Attachment Theory: John Bowlby and Mary Ainsworth. Developmental Psychology, 28(5), 759-75. https://doi.org/10.1037/0012-1649.28.5.759

Bowlby, J. (1982). Attachment and loss (2nd ed.). New York: Basic Books.

Brussoni, M., Jang, K., Livesley, W., \& Macbeth, T. (2000). Genetic and environmental influences on adult attachment styles. Personal Relationships, 7(3), 283-289.

Chang, J., Wang, S., Mancini, C., Mcgrath-Mahrer, B., \& Orama de Jesus, S. (2019). The complexity of cultural mismatch in higher education: Norms affecting first-generation college students' coping and help-seeking behaviors. Cultural Diversity \& Ethnic Minority Psychology, Cultural diversity \& ethnic minority psychology, 14 October 2019.

Chapman, T., \& Bhopal, K. (2019). The perils of integration: Exploring the experiences of African American and black Caribbean students in predominantly white secondary schools. Ethnic and Racial Studies, 42(7), 1110-1129. 
Crawshaw, J., \& Game, A. (2015). The role of line managers in employee career management: An attachment theory perspective. The International Journal of Human Resource Management, 26(9), 1182-1203.

Davila, J., Burge, D., \& Hammen, C. (1997). Why does attachment style change? Journal of Personality and Social Psychology, 73(4), 826-838.

Davila, J., \& Sargent, E. (2003). The Meaning of Life (Events) Predicts Changes in Attachment Security. Personality and Social Psychology Bulletin, 29(11), 1383-1395.

Hiester, M., Nordstrom, A., \& Swenson, L.M. (2009). Stability and Change in Parental Attachment and Adjustment Outcomes During the First Semester Transition to College Life. Journal of College Student Development 50(5), 521-538. doi:10.1353/csd.0.0089.

Judge, Timothy A., \& Watanabe, Shinichiro. (1993). Another look at the job satisfaction-life satisfaction relationship. Journal of Applied Psychology, 78(6), 939-948.

Kafetsios, K., Athanasiadou, M., \& Dimou, N. (2014). Leaders' and subordinates' attachment orientations, emotion regulation capabilities and affect at work: A multilevel analysis. The Leadership Quarterly, 25(3), 512-527.

Keller, H. (2013). Attachment and Culture. Journal of Cross-Cultural Psychology, 44(2), 175-194. Keller, T., \& Cacioppe, R. (2001). Leader-follower attachments: Understanding parental images at work. Leadership \& Organization Development Journal, 22(2), 70-75.

Kirschner, S., \& Martin, Jack. (2010). The sociocultural turn in psychology : The contextual emergence of mind and self. New York: Columbia University Press.

Kobak, R., \& Sceery, A. (1988). Attachment in Late Adolescence: Working Models, Affect Regulation, and Representations of Self and Others. Child Development, 59(1), 135-46. 
Konrath, S. H., Chopik, W. J., Hsing, C. K., \& O’brien, E. (2014). Changes in Adult Attachment Styles in American College Students Over Time: A Meta-Analysis. Personality and Social Psychology Review, 18(4), 326-348. https://doi.org/10.1177/1088868314530516

Lavy, S. (2017). Who benefits from group work in higher education? An attachment theory perspective. Higher Education, 73(2), 175-187.

Little, L., Nelson, D., Wallace, J., \& Johnson, P. (2011). Integrating attachment style, vigor at work, and extra-role performance. Journal of Organizational Behavior, 32(3), 464-484.

Lopez, F. G. (1997). Student-Professor Relationship Styles, Childhood Attachment Bonds and Current Academic Orientations. Journal of Social and Personal Relationships, 14(2), 271-282. https://doi.org/10.1177/0265407597142008

Lopez, F. G., \& Gormley, B. (2002). Stability and change in adult attachment style over the first-year college transition: Relations to self-confidence, coping, and distress patterns. Journal of Counseling Psychology, 49(3), 355-364. http://dx.doi.org/10.1037/0022-0167.49.3.355

Landen, S. M., \& Wang, C.-C. D. C. (2010). Adult attachment, work cohesion, coping, and psychological well-being of firefighters. Counselling Psychology Quarterly, 23(2), 143-162. https://doi.org/10.1080/09515071003776028

Mikulincer, M. (2007). Attachment in adulthood: Structure, dynamics, and change. Retrieved from http://public.eblib.com/choice/publicfullrecord.aspx? $\mathrm{p}=320570$

Mischel, W. (2004). Toward an integrative science of the person. Annual Review of Psychology, 55, 1. 
Molero, F., Moriano, J., \& Shaver, P. (2013). The influence of leadership style on subordinates' attachment to the leader. The Spanish Journal of Psychology, 16, E62.

Nadal, K., Griffin, K., Wong, Y., Hamit, S., \& Rasmus, M. (2014). The Impact of Racial Microaggressions on Mental Health: Counseling Implications for Clients of Color. Journal of Counseling \& Development, 92(1), 57-66.

Ognibene, T., \& Collins, N. (1998). Adult Attachment Styles, Perceived Social Support and Coping Strategies. Journal of Social and Personal Relationships, 15(3), 323-345.

Parade, S., Leerkes, E., \& Blankson, A. (2010). Attachment to parents, social anxiety, and close relationships of female students over the transition to college. Journal of Youth and Adolescence, $39(2), 127-137$.

Ravitz, P., Maunder, R., Hunter, J., Sthankiya, B., \& Lancee, W. (2010). Adult attachment measures: A 25-year review. Journal of Psychosomatic Research, 69(4), 419-432.

Richards, D., \& Schat, A. (2011). Attachment at (not to) work: Applying attachment theory to explain individual behavior in organizations. The Journal of Applied Psychology, 96(1), 169-182.

Roisman, G., Padron, E., Sroufe, L., \& Egeland, B. (2002). Earned-Secure Attachment Status in Retrospect and Prospect. Child Development, 73(4), 1204-1219.

Simatele, M. (2018). A Cross-Cultural Experience of Microaggression in Academia: A Personal Reflection. Education as Change, 22(3), Education as Change, 2018, Vol.22(3).

Tinto, V. (1990). Principles of Effective Retention. Journal of the Freshman Year Experience, 2(1), $35-48$. 
Underwood, R., Mohr, D., \& Ross, M. (2016). Attachment Style, Leadership Behavior, and Perceptions of Leader Effectiveness in Academic Management. Journal of Leadership Education, 15(4), 100-116.

Vogel, D., \& Wei, M. (2005). Adult Attachment and Help-Seeking Intent: The Mediating Roles of Psychological Distress and Perceived Social Support. Journal of Counseling Psychology, 52(3), 347-357. 